\title{
A case of a leukocytosis diagnosed as chronic myeloid leukemia on a health checkup
}

\author{
Sayaka KaWAno $^{1} \quad$ Johji Kato $^{2}$ Kazuo Kitamura $^{1}$ \\ ${ }^{I}$ Department of Internal Medicine, Circulatory and Body Fluid Regulation, Faculty of Medicine, University of Miyazaki \\ ${ }^{2}$ Frontier Science Research Center, University of Miyazaki
}

\begin{abstract}
Leukocytosis is reported to have malignant and non-malignant etiologies such as infectious diseases and smoking. However, it is important to rule out asymptomatic cases of hematological malignancy in the early stages based on leukocytosis findings on blood tests during health checkups. Here, we report a 52-year-old asymptomatic woman with leukocytosis (white blood cell count of $13.01 \times 10^{9} / \mathrm{L}$ ) that was incidentally detected on a blood test performed as part of a routine health checkup. According to the diagnostic criteria of the Japan Society of Ningen Dock, the examinee was referred to another regional hospital for further evaluation. Thereafter, based on findings from blood smears, bone marrow examination and major BCR/ABL polymerase chain reaction (PCR) examination, the patient was clinically diagnosed with chronic myeloid leukemia. Consequently, diagnosis during the early stages allowed successful treatment with a tyrosine kinase inhibitor, and the patient became disease-free.

In conclusion, when we diagnose the leukocytosis on the blood test in health checkups, we should consult and refer to specialists to perform further detailed examinations and therapy in clinical practice.

(HEP. 2020; 47: 523-526.)
\end{abstract}

Key words leukocytosis, repeat complete blood count, peripheral blood smear, health checkup, chronic myeloid leukemia

\section{Introduction}

Leukocytosis $^{1)}$ is reported to have various etiologies, including hematological malignancies such as chronic myeloid leukemia, acute myeloid leukemia, chronic lymphoid leukemia, and myelofibrosis $^{2)}$, and non-malignant etiologies such as infectious diseases, smoking, obesity, and pregnancy ${ }^{2}$. Of these etiologies, it is essential to rule out asymptomatic cases of hematological malignancy during the early stages based on leukocytosis findings on blood tests during health checkups.

Although previous reports are limited, it has been suggested that mild leukocytosis is usually asymptomatic and is occasionally diagnosed based on blood test findings during health check$u^{3} s^{3)}$. In one study, 13 of 2,182 patients who underwent health checkups were diagnosed with leukocytosis, and 2 of 6 were diagnosed with leukemia $(0.1 \%)^{3)}$.

Here, we report a woman who presented with leukocytosis that was incidentally diagnosed on a blood test during a health checkup.

\section{Case report}

A 52-year-old asymptomatic woman, who was a non-smoker, underwent an annual health checkup. The medical history of the examinee was taken by nurses using a questionnaire, which was

\footnotetext{
Received: August 2, 2019, Accepted: September 27, 2019

Corresponding author: Sayaka Kawano

${ }^{1}$ Address; University of Miyazaki

5200 Kihara, Kiyotake, Miyazaki 889-1692, Japan

TEL: +81-985-85-1510

E-mail: sayakawano23@yahoo.co.jp
}

confirmed by a physician. The questionnaire consisted of the chief complaint, medical history, smoking status, medication use, and family history of disease. The examinee had no cough, sore throat, fever, and significant medical and family history of disease. The examinee had a body mass index of $24.5 \mathrm{~kg} / \mathrm{m}^{2}$. Physical examination, including auscultation, revealed that the respiratory sound was clear and heart sound had no murmur. The hepatosplenomegaly was not palpable. The chest X-ray revealed normal findings with no signs of acute bronchitis and pneumonia. The electrocardiogram showed normal sinus rhythm and no ST-T change. Double-contrast upper gastrointestinal barium X-ray radiography, mammography, cervical uterine cancer screening, and urinary examination findings were normal. The health checkup findings were normal, except for leukocytosis (white blood cell count of $13.01 \times 10^{9} / \mathrm{L}$ ). Previous medical records were consulted to determine the onset of leukocytosis, but it was not detected during previous checkups. An increase in the white blood cell count $\left(13.01 \times 10^{9} / \mathrm{L}\right)$ was incidentally identified on a blood test performed as part of the checkup. According to the diagnostic criteria of the Japan Society of Ningen Dock ${ }^{1}$, a further detailed examination was recommended, judged as D2 criteria, for which the examinee was referred to the Department of Internal Medicine at our institution. Repeat complete blood count (CBC) examinations revealed continuous leukocytosis over two months. The serial change in white blood cell count is detailed in Table 1. Subsequently, the patient was referred to the Department of Hematology at the regional hospital for further examinations. The physical examination on admission revealed that the patient was normotensive with a normal sinus rhythm. Laboratory findings (Table 2) revealed leukocytosis (white blood cell count of 
Table 1 Transition of leukocytosis to CML before and after the diagnosis and CML treatment between 2008 and 2018

\begin{tabular}{|c|c|c|c|c|c|c|c|c|c|c|c|c|}
\hline & $\begin{array}{c}2008 \\
6 / 5\end{array}$ & $\begin{array}{l}2009 \\
6 / 4\end{array}$ & $\begin{array}{l}2010 \\
6 / 3\end{array}$ & $\begin{array}{l}2010 \\
6 / 18\end{array}$ & $\begin{array}{l}2010 \\
7 / 16\end{array}$ & $\begin{array}{l}2010 \\
7 / 30\end{array}$ & $\begin{array}{l}2010 \\
8 / 10\end{array}$ & $\begin{array}{l}2010 \\
8 / 10\end{array}$ & $\begin{array}{c}2012 \\
9 / 11\end{array}$ & $\begin{array}{l}2014 \\
8 / 26\end{array}$ & $\begin{array}{l}2016 \\
9 / 20\end{array}$ & $\begin{array}{l}2018 \\
11 / 1\end{array}$ \\
\hline & \multicolumn{4}{|c|}{ Medical health checkup } & \multicolumn{3}{|c|}{$\begin{array}{l}\text { Department of } \\
\text { Internal medicine }\end{array}$} & $\begin{array}{c}\text { Department of } \\
\text { Hematology }\end{array}$ & \multicolumn{4}{|c|}{ Medical health checkup } \\
\hline WBC $\left(\times 10^{9} / L\right)$ & 5.87 & 6.63 & 13.01 & 16.20 & 11.30 & 11.90 & 15.70 & 13.32 & 3.30 & 3.92 & 3.39 & 3.40 \\
\hline Neutrophil (\%) & 51.4 & n.e. & n.e. & n.e. & n.a. & n.a. & n.a. & 64.5 & n.e. & n.e. & n.e. & 44.7 \\
\hline Lymphocyte (\%) & 39.9 & n.e. & n.e. & n.e. & n.a. & n.a. & n.a. & 17.0 & n.e. & n.e. & n.e. & 44.1 \\
\hline Monocyte (\%) & 5.8 & n.e. & n.e. & n.e. & n.a. & n.a. & n.a. & 4.0 & n.e. & n.e. & n.e. & 7.9 \\
\hline Eosinophil (\%) & 1.9 & n.e. & n.e. & n.e. & n.a. & n.a. & n.a. & 1.5 & n.e. & n.e. & n.e. & 2.4 \\
\hline \multirow[t]{4}{*}{ Basophil (\%) } & 1.0 & n.e. & n.e. & n.e. & n.a. & n.a. & n.a. & 6.0 & n.e. & n.e. & n.e. & 0.9 \\
\hline & & & & & & & & Myelocyte $2.0 \%$ & & & & \\
\hline & & & & & & & & Metamyelocyte $5.0 \%$ & & & & \\
\hline & & & & & & & & Blast $0.0 \%$ & & & & \\
\hline $\mathrm{Hb}(\mathrm{g} / \mathrm{dL})$ & 13.4 & 13.7 & 14.1 & 14.7 & n.a. & n.a. & n.a. & 13.5 & 11.1 & 11.9 & 10.7 & 11.4 \\
\hline Plt $\left(\times 10^{9} / L\right)$ & 24.5 & n.e. & n.e. & 28.9 & n.a. & n.a. & n.a. & 23.7 & n.e. & n.e. & n.e. & n.e. \\
\hline
\end{tabular}

abbrebiations: n.e; not examined, n.a; not available

Table 2 Laboratory findings on admission

\begin{tabular}{|c|c|c|c|c|c|c|c|c|}
\hline WBC & 13.32 & $\times 10^{9} / \mathrm{L}$ & & & & & & \\
\hline Promyelocyte & 0.0 & $\%$ & T.Bil & 0.6 & $\mathrm{mg} / \mathrm{dL}$ & PT & 74.4 & $\%$ \\
\hline Myelocyte & 2.0 & $\%$ & AST & 28 & $\mathrm{IU} / \mathrm{L}$ & PT-INR & 1.21 & \\
\hline Metamyelocyte & 5.0 & $\%$ & ALT & 29 & $\mathrm{IU} / \mathrm{L}$ & APTT & 30.8 & $\mathrm{sec}$ \\
\hline Neutrophil-Stab & 5.5 & $\%$ & ALP & 200 & $\mathrm{IU} / \mathrm{L}$ & $\mathrm{Fbg}$ & 279.7 & $\mathrm{mg} / \mathrm{dL}$ \\
\hline Neutrophil-Seg & 59.0 & $\%$ & LDH & 429 & $\mathrm{IU} / \mathrm{L}$ & FDP & 1 & $\mu \mathrm{g} / \mathrm{mL}$ \\
\hline Lymphocyte & 17.0 & $\%$ & CK & 58 & $I U / L$ & & & \\
\hline Monocyte & 4.0 & $\%$ & $\mathrm{Na}$ & 140 & $\mathrm{mEq} / \mathrm{dL}$ & NAP score & 55 & $\%(75-95)$ \\
\hline Eosinophil & 1.5 & $\%$ & $\mathrm{~K}$ & 3.6 & $\mathrm{mEq} / \mathrm{dL}$ & Vit B12 & 1,000 & $\mathrm{pg} / \mathrm{mL}$ \\
\hline Basophil & 6.0 & $\%$ & $\mathrm{Cl}$ & 103 & $\mathrm{mEq} / \mathrm{dL}$ & & & \\
\hline Blast & 0.0 & $\%$ & $\mathrm{Ca}$ & 9.0 & $\mathrm{mg} / \mathrm{dL}$ & & & \\
\hline RBC & 4.32 & $\times 10^{12} / \mathrm{L}$ & BUN & 7.3 & $\mathrm{mg} / \mathrm{dL}$ & & & \\
\hline $\mathrm{Hb}$ & 13.5 & $\mathrm{~g} / \mathrm{dL}$ & CRE & 0.6 & $\mathrm{mg} / \mathrm{dL}$ & & & \\
\hline $\mathrm{Ht}$ & 40.7 & $\%$ & UA & 4.3 & $\mathrm{mg} / \mathrm{dL}$ & & & \\
\hline MCV & 94.2 & $f L$ & Ferri & 44 & $\mathrm{ng} / \mathrm{mL}$ & & & \\
\hline $\mathrm{MCHC}$ & 33.2 & $\%$ & TP & 7.7 & $\mathrm{~g} / \mathrm{dL}$ & & & \\
\hline Plt & 237 & $\times 10^{9} / \mathrm{L}$ & ALB & 4.6 & $\mathrm{~g} / \mathrm{dL}$ & & & \\
\hline Ret & 1.8 & $\%$ & CRP & 0.03 & $\mathrm{mg} / \mathrm{dL}$ & & & \\
\hline
\end{tabular}

$13.32 \times 10 \%$ L, with $2.0 \%$ myelocytes, $5.0 \%$ metamyelocytes, $5.5 \%$ neutrophils-stab, $59.0 \%$ neutrophils-seg, $17.0 \%$ lymphocytes, $4.0 \%$ monocytes, $1.5 \%$ eosinophils, and $6.0 \%$ basophils) and an elevated serum lactate dehydrogenase level (429 IU/L). Thus, leukocytosis was also observed on the repeat examination. Furthermore, a peripheral blood smear examination revealed an increase in basophils. Since the peripheral blood test findings were abnormal, additional tests such as bone marrow examination were required. Bone marrow examination revealed myeloid hyperplasia, with a blast percentage of $0.6 \%$. The bone marrow smear is shown in Figure 1. The positive finding of Major bcr/ abl Fluorescence in situ hybridization (FISH) and polymerase chain reaction (PCR) analysis strongly supported a chronic myeloid leukemia with chronic phase (Fig. 2). Consequently, a final diagnosis of chronic-phase chronic myeloid leukemia was made. According to the guidelines for the treatment of chronic myeloid leukemia ${ }^{4,5}$, imatinib (an inhibitor of BCR/ABL) was administered. Because of adverse effects on the skin, imatinib was discontinued and dasatinib was administered. Consequently, the patient achieved complete molecular remission, survived, and became disease-free. Thereafter, the patient underwent an annual health checkup after CML diagnosis and therapy. 


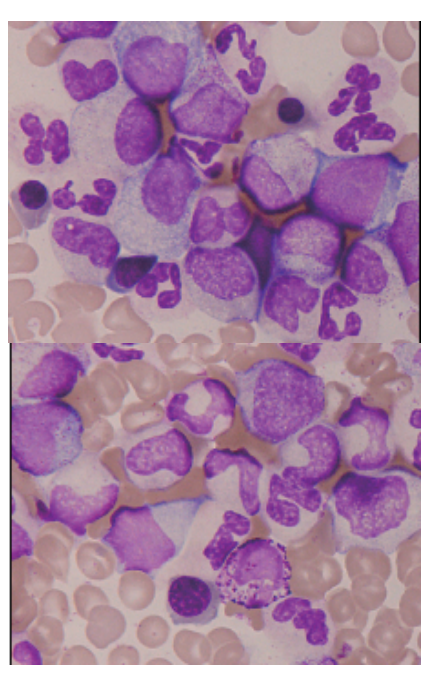

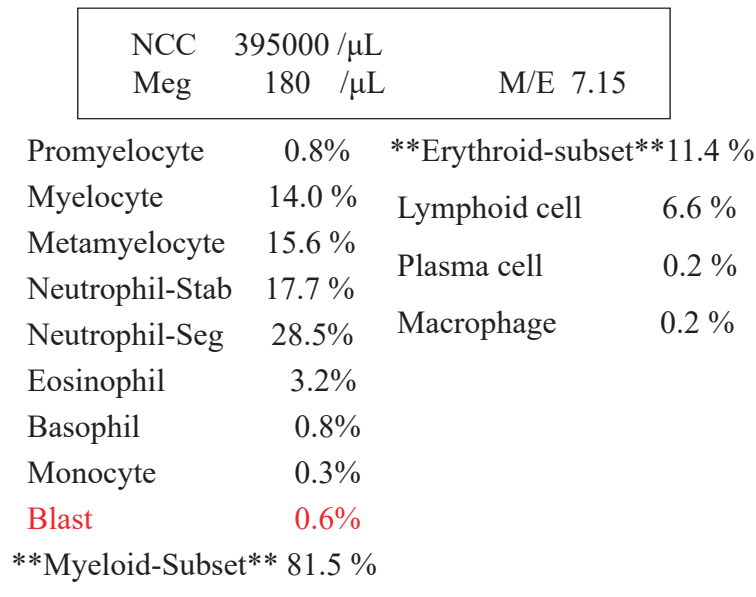

Fig. 1 The results from bone marrow examination

\section{A. Chromosomal anaylsis}

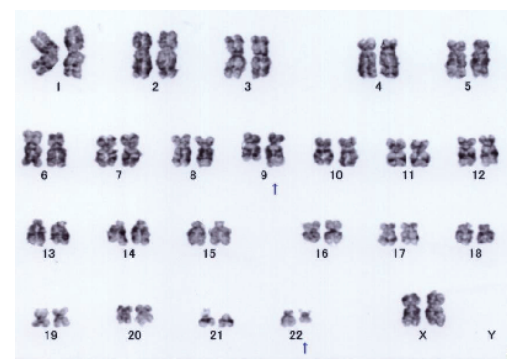

B. FISH analysis

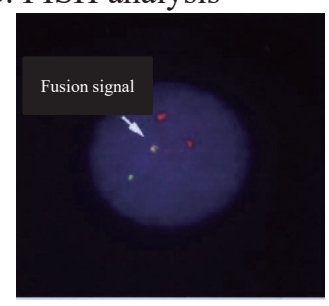

Fig. 2 Major bcr/abl FISH and PCR analysis

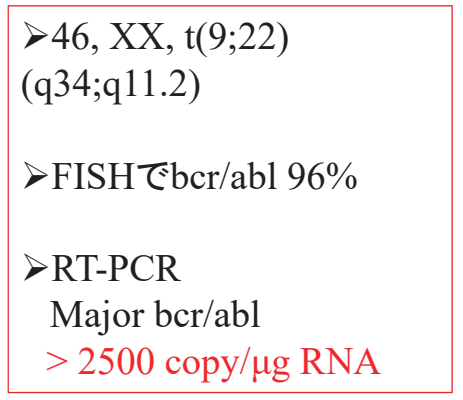

\section{Discussion}

We describe an asymptomatic case of chronic myeloid leukemia presenting with leukocytosis on a health checkup that was finally diagnosed after detailed examination in a regional hospital.

First, regarding as the etiology of leukocytosis, hematological malignancy may be rarely included and should be ruled out in clinical practice. Riley et al. reported that leukocytosis may herald a malignant disorder, such as acute or chronic leukemia, or a myeloproliferative disorder, such as polycythemia vera, myelofibrosis, or essential thrombocytosis ${ }^{2)}$. Among these, chronic leukemias are most commonly diagnosed after incidental findings on complete blood counts in asymptomatic patients ${ }^{2}$. Similarly, in our case, leukocytosis was incidentally detected on the blood test performed as part of a health checkup. The etiologies of leukocy- tosis varied from benign disorders, such as smoking and metabolic syndrome, to hematological malignancy ${ }^{2,6,7)}$. For the benign disorders, the examinees did not need to undergo further examinations. However, for the hematological malignancy, the examinees required urgent further examinations. Due to the limited examinations included in health checkups and varied etiologies of leukocytosis, it is difficult for our health checkup team to convey the accurate etiology and diagnosis of leukocytosis.

Therefore, when the white blood cell count was over $10.0 \times$ $10^{9} / \mathrm{L}$, we assessed it as D2 criteria according to the diagnostic criteria of the Japan Society of Ningen Dock ${ }^{1)}$ and recommended further detailed examinations. Thus, we should consult and refer to the specialists to perform additional assessments such as repeat complete blood count and peripheral blood smear tests.

Second, regarding the usefulness of medical health checkups in leukocytosis, a combination of information such as past medi- 
cal history, life history, physical examination findings, and peripheral blood cells counts is essential to rule out the various etiologies of leukocytosis.

Although an extensive PubMeb search revealed limited studies on this topic, in a study by Takahashi et al., 13 of 2,182 patients who underwent a health checkup were diagnosed with leukocytosis. The suspected cause of leukocytosis was smoking in 6 patients, transient infection in 4, and unknown in 1; moreover, 2 of the 13 patients were diagnosed with leukemia $(0.1 \%)^{3}$. Of the total 10,412 health checkup examinees in 2010 at our health checkups institution, only one case was associated with CML.

Riley et al. reported that the normal range for white blood cell counts changes with age and pregnancy ${ }^{2}$. These factors should be considered while diagnosing the etiology of leukocytosis. Obtaining additional information, such as the history of smoking, medication, pregnancy, and splenectomy, is also important ${ }^{2}$. Our patient was a non-smoker and had no history of splenectomy. Furthermore, an algorithm for the evaluation of leukocytosis is recommended for repeat complete blood count and peripheral smear examination ${ }^{2}$. According to this algorithm, our patient presented with leukocytosis on repeat examination and detailed examination in a regional hospital, revealing that the patient had chronic myeloid leukemia.

In conclusion, when leukocytosis is identified during health checkups, we should consult and refer to the specialists to perform further detailed examination, to elucidate the etiology of leukocytosis.

\section{Compliance and ethics standards}

Ethics statement: This case study was conducted in compliance with good clinical practices and the ethics principles of the Declaration of Helsinki.

Informed consent: Informed consent was obtained from the patient for reporting these case details.

\section{The authors state that they have no Conflict of Interest (COI).}

\section{REFERENCES}

1) Japan Society of Ningen Dock. The diagnostic criteria of 2019. Tokyo: 2019.

2) Riley LK, Rupert J. Evaluation of Patients with Leukocytosis. Am Fam Physician 2015; 92(11): 1004-11.

3) Takahashi Y, Izumi N, Kato A. Investigation on Subjects with Leukocytosis Discovered in Health Check-ups at Our Hospital. Ningen Dock 2013; 28(1): 43-8.

4) Usui N. JSH guideline for tumors of hematopoietic and lymphoid tissues-leukemia: 4. Chronic myelogenous leukemia (CML)/ myeloproliferative neoplasms (MPN). Int J Hematol 2017; 106(5): 591-611.

5) Baccarani M, Castagnetti F, Gugliotta G, Rosti G. A review of the European Leukemia Net recommendations for the management of CML. Ann Hematol 2015; 94(Suppl 2): S141-7.

6) Nagasawa N, Tamakoshi K, Yatsuya H, Hori Y, Ishikawa M, Murata $\mathrm{C}$, et al. Association of white blood cell count and clustered components of metabolic syndrome in Japanese men. Circ J 2004; 68(10): 892-7.

7) Kurata C. Medical check-up findings characteristic of smokers: aimed at improving smoking cessation interventions by physicians. Intern Med 2006; 45(18): 1027-32. Epub 2006 Oct 16. 